\title{
The threaded molecular clumps of Chamaeleon III
}

\author{
G. F. Gahm ${ }^{1}$, K. Lehtinen ${ }^{2}$, P. Carlqvist ${ }^{3}$, J. Harju², M. Juvela ${ }^{2}$, and K. Mattila² \\ 1 SCFAB, Stockholm Observatory, 10691 Stockholm, Sweden \\ 2 Observatory, PO Box 14, 00014 University of Helsinki, Finland \\ 3 Alfvén Laboratory, Royal Institute of Technology, 10044 Stockholm, Sweden
}

Received 16 October 2001 / Accepted 22 March 2002

\begin{abstract}
We have mapped large areas in the Chamaeleon III complex of molecular clouds with the SEST in ${ }^{13} \mathrm{CO}(J=1-0)$ and in $\mathrm{C}^{18} \mathrm{O}(J=1-0)$. The stronger $\mathrm{CO}$ emission coincides with areas of cold dust emission, which is distributed in long, but thin, wavy filaments. We identify some 40 clumps of enhanced CO emission in these filaments. In the southern part of Cha III the clumps are equidistant along the main zig-zag shaped filament. Here we find two systems of filaments moving at different radial velocities. At least part of the zig-zag patterns visible on optical images may be caused by overlapping filaments. All clumps are small (typically 0.02-0.05 pc in radius), and of small mass (typically $0.1-0.7 M_{\odot}$, when assuming the "standard" $\mathrm{C}^{18} \mathrm{O} / \mathrm{H}_{2}$ column density ratio). Also the average number densities are small, $n\left(\mathrm{H}_{2}\right)=1 \times 10^{4}-8 \times 10^{4} \mathrm{~cm}^{-3}$, and the density contrast between clump and interclump gas is only $\sim 10$. In addition the values of $\left|E_{\text {pot }}\right| / E_{\text {kin }}$ are unusually small, $0.03-0.33$. These clumps have smaller masses than those so far identified in other molecular clouds. Previously reported clumps of larger masses in Cha III turn out to be composed of assemblies of clumps. There are no signs of star formation in Cha III (unlike Cha I and Cha II), and our results indicate also that such activity is not expected. However, with the velocity dispersion of $0.2 \mathrm{~km} \mathrm{~s}^{-1}$ the clumps would leave the thin filaments on short timescales, and if the clumps as such are not confined by some external force, they would also lose their identity on even shorter timescales. We discuss the possibility that the clumps are confined by electromagnetic forces, and show that this may work with reasonable assumptions on the required magnetic field strength. We also discuss the possibility that the clumps are attached to magnetic ropes along the filamentary axis, in which case the clumps could swing back and forth perpendicularly to the axis, like they were threaded on elastic strings.
\end{abstract}

Key words. ISM: clouds - ISM: structure

\section{Introduction}

Many interstellar molecular clouds contain elongated structures. These stuctures can show wavy, and even sinusoidal shapes. Such forms are apparent on the very large scales, as seen on sky maps of Hi (Hartmann \& Burton 1997; Verschuur 1991), and as building blocks in complexes like the Orion GMC (see e.g. Bally 1989) and the Taurus clouds (Mizuno et al. 1995). Extremely thin but extended, dark, wavy filaments are present in many nebular regions, for instance the Lagoon nebula (Caulet 1997) and the Rosette nebula (Carlqvist et al. 1998). Molecular clouds are also composed of clumps of various size and mass. Clump mass spectra of molecular clouds are consistent with power laws of the type $\mathrm{d} N / \mathrm{d} M \propto M^{-\alpha}$, with $\alpha$ between 1.4 and 2 (e.g. Elmegreen \& Falgarone 1996; Kramer et al. 1998).

Send offprint requests to: G. F. Gahm,

e-mail: gahm@astro.su.se

* Based on observations made with the Swedish ESO Submillimetre Telescope (SEST), Chile.
The architecture of individual clouds is linked to activities in the environment, but also to internal forces produced for instance by star formation in the clouds. Magnetic fields of some ten $\mu \mathrm{G}$ have been measured by methods relying on Zeeman splitting (e.g. Crutcher 1999), and influence the physics of the clouds. Under certain conditions the magnetic fields may dominate the dynamics and evolution of individual clouds (e.g. Lazarian 1993; Nakamura et al. 1995; Gehman et al. 1996; Nakajima \& Hanawa 1996; Padoan et al. 1998; Nagai et al. 1998; Fiege \& Pudritz 2000b). In addition, filaments with sinusoidal or serpentine-like shapes may point at the presence of twisted, helical structures which are associated to electric currents, as discussed e.g. by Bally (1989), Shibata \& Matsumoto (1991), Ushida et al. (1991), Carlqvist \& Gahm (1992), Hanawa et al. (1993), Verschuur (1995), Cox (1996), Fiege \& Pudritz (2000a), Falgarone et al. (2001) and Carlqvist et al. (2001).

By selecting filamentary clouds lacking signs of advanced star formation, one can better study the effects of magnetic fields on cloud evolution. Here, we present CO observations of such a complex of dark, wavy, filamentary 


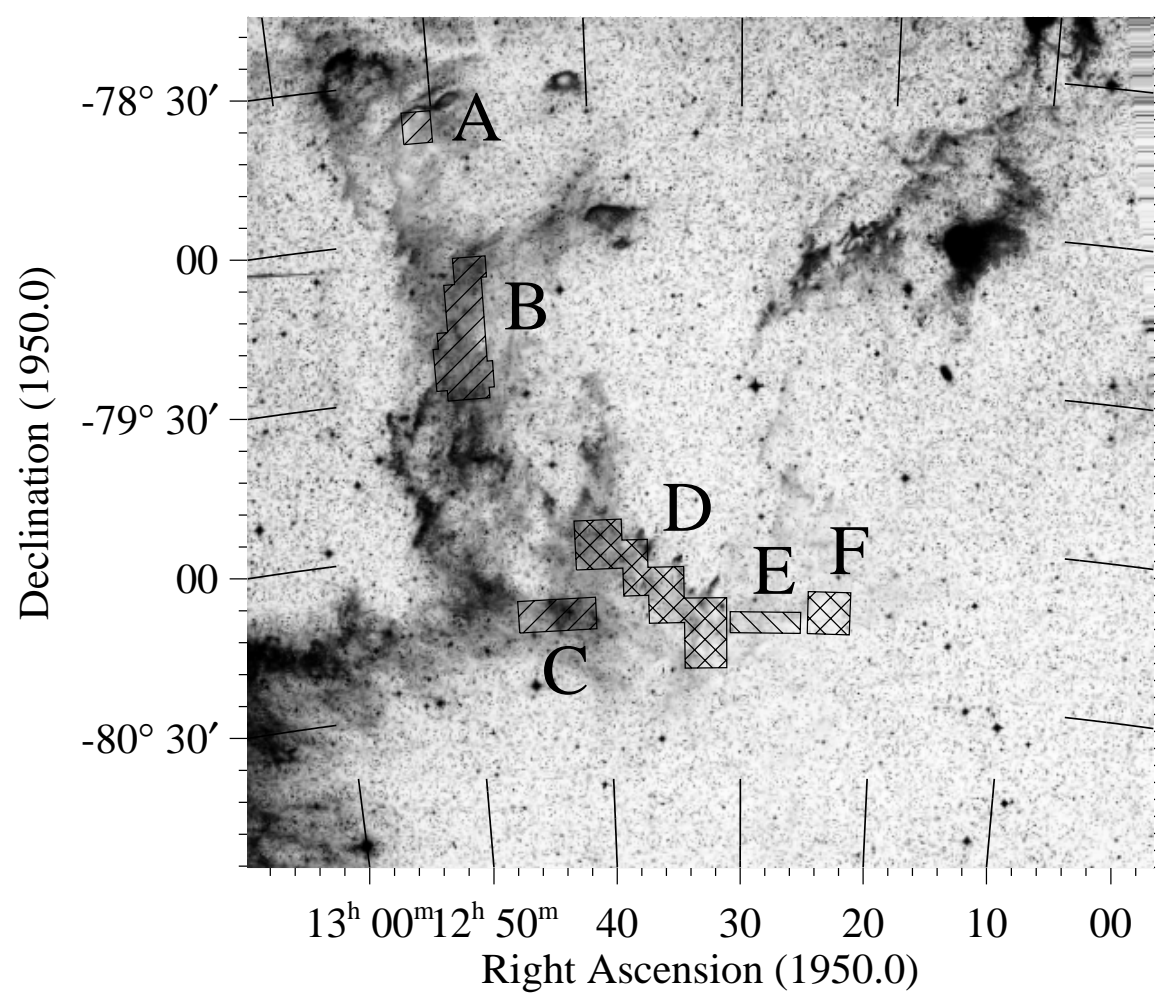

Fig. 1. Dark clouds with associated reflection nebulosities are apparent on this red ESO Schmidt plate covering the Chamaeleon III complex. The nebulosities in the upper right of the image are related to DC300-17, which may form a separate complex. The distinct dark core at $\alpha=12^{\mathrm{h}} 41^{\mathrm{m}}, \delta=-78^{\circ} 32^{\prime}$ is the so called "Thumbprint". The regions that were observed for molecular line emission are labeled from $\mathrm{A}$ to $\mathrm{F}$. The filaments extending over regions $\mathrm{C}$ to $\mathrm{F}$ are called the Serpentine in the text, with the tilted filament in region D. Regions A and B were observed primarily in $\mathrm{C}^{18} \mathrm{O}$, and region $\mathrm{E}$ only in ${ }^{13} \mathrm{CO}$, while the rest of the Serpentine was observed at both frequencies.

clouds, namely Chamaeleon III which is part of the very large association of dark clouds in Chamaeleon at intermediate galactic latitude $\left(b^{\mathrm{II}} \sim-15^{\circ}\right)$. We were stimulated to make this study by the unusual forms, serpentine- or zig-zag-patterns, which are seen especially in the southern dark filaments of Cha III.

\section{Chamaeleon III - a description}

Cha III extends over about $3^{\circ} \times 3^{\circ}$ in the southern part of the large complex with connections to Cha II in the north. The distribution of obscuring dust clouds in the area has been illustrated by Feitzinger \& Stüwe (1984), and more detailed extinction maps have been presented by Hetem et al. (1988) and Cambrésy (1999). To the east is the cloud DC300-17 (Hartley et al. 1986), which could form a separate structure, yet associated to the Chamaeleon complex. Several estimates of the distance to Cha III converge at values 150 pc (Franco 1991; Whittet et al. 1997; Corradi et al. 1997; Knude \& Høg 1998).

The ESO/SERC Schmidt plates show a complex pattern of dark knots connected by elongated, dark, wavy filaments, which at the southern part of Cha III show a serpentine-like shape. Bright rims with finger-like extensions are apparent, and a web of very faint, extremely thin but very long and straight shining filaments. These feeble

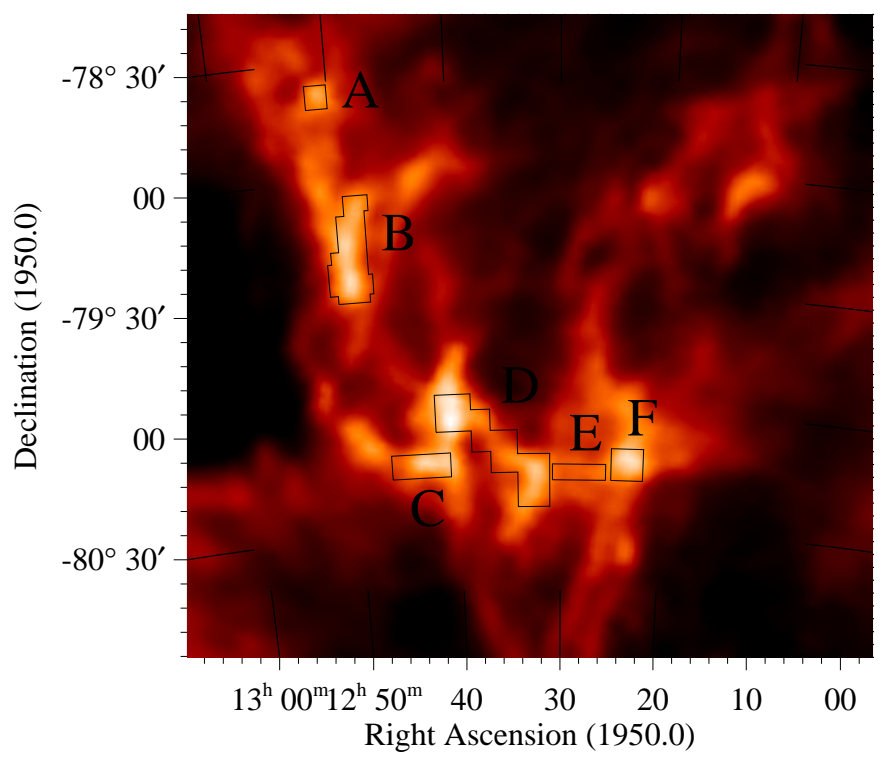

Fig. 2. IRAS map of the $100 \mu \mathrm{m}$ emission in the same area as shown in Fig. 1. A system of interwoven filaments is present. Filaments cross each other at certain locations.

structures, reflecting stellar light, extend over the entire Chamaeleon complex. 
The few pre-main-sequence candidates found from surveys of the Cha III region are all located outside the major molecular filaments in the area (Schwartz 1991; Hetem et al. 1988; Alcala et al. 1995; Feigelson 1996; Nordh et al. 1996; Alcala et al. 1997; Covino et al. 1997). Pfau et al. (1996) announced a number of $\mathrm{H} \alpha$ objects in Cha III. Most of these are fainter than magnitude 16 in $B$ and located outside areas of major obscuration (Pfau 2000). T Tauri stars at these distances and obscurations would have typically $B<15$. A few of the brighter objects lie close to the cloud boundaries, and these stars should be checked in more detail. Hence, with some reservation, the system of elongated, whispy and serpentine-like dark clouds appears to be juvenile - no signs that star formation has yet advanced very far.

Practically the entire Cha III complex was mapped in ${ }^{13} \mathrm{CO}$ by Mizuno et al. (1998) and $\mathrm{C}^{18} \mathrm{O}$ by Mizuno et al. (1999) with an $8^{\prime}$ spacing. The authors identify 6 dense molecular cores in Cha III in the mass range 6-30 $M_{\odot}$, well below the corresponding virial masses. Most of this area was also observed in ${ }^{12} \mathrm{CO}$ by Boulanger et al. (1998) with a somewhat smaller spacing. A very detailed map of the infrared emission from cold and warm dust, based on images from IRAS (Infrared Astronomical Satellite), was presented. This image very nicely shows how the dust is distributed in bundles of interwoven threads forming the filaments in the denser regions of Cha III. Finally, Vilas-Boas et al. (1994) made pointed observations in ${ }^{13} \mathrm{CO}$ and $\mathrm{C}^{18} \mathrm{O}$ of optically selected cores with sizes of a few arcmin.

The main part of the molecular clouds in Cha III is confined to an area with a projected size of radius $4 \mathrm{pc}$ containing of the order of $10^{3} M_{\odot}$ (Boulanger et al. 1998). The complex is linked to other cloud complexes like the Southern Coalsack and the Musca dark clouds, possibly forming an interface between the local bubble and Loop I superbubbles (Corradi et al. 1997). In addition, Eggen (1998) has proposed a link to the Sco OB2 supercluster. Finally, the Chamaeleon cloud complex is engulfed in high-velocity gas moving with velocities up to $50 \mathrm{~km} \mathrm{~s}^{-1}$ relative to the complex. The origin of this gas component is unknown, but has been related to supernova driven bubbles or the interaction of infalling gas with gas in the disk (Cleary et al. 1979; Gry et al. 1998).

\section{Observations}

Figure 1 is selected from our $180 \mathrm{~min}$ exposure obtained with the ESO Schmidt telescope in 1994 on a Kodak IIIaF photographic plate in combination with an RG630 filter, providing a deep, red image of Cha III and surroundings. Inserted are the areas observed for molecular emission, which are labeled from A to F. To the right of area Cha III-A are globule-like extensions. One of these is the "Thumbprint" nebula, investigated earlier for molecular line emission by Lehtinen et al. (1995). The serpentinelike feature extends through areas Cha III-C to Cha III-F ( $\mathrm{C}$ to $\mathrm{F}$ in the figure), and will be referred to as the
Serpentine below. Note the finger-like extensions of dust pointing north-west from the tilted filament (Cha III-D). The faint shining nebulosities seen here, and in other parts of the complex, are the result of scattered stellar light. Figure 2 shows the IRAS $100 \mu \mathrm{m}$ emission from dust in the same area as Fig. 1.

The main body of the molecular line observations were carried out in 1992 with the SEST equipped with a cooled Schottky diode mixer receiver, and a 2000 channel acoustic-optic spectrometer (AOS). The bandwidth of the spectrometer was $86 \mathrm{MHz}$ and the resulting channel separation was $0.12 \mathrm{~km} \mathrm{~s}^{-1}$ at $110 \mathrm{GHz}$. The observed transitions were ${ }^{13} \mathrm{CO}(J=1-0), \mathrm{C}^{18} \mathrm{O}(J=1-0)$, $\mathrm{HCN}(J=1-0), \operatorname{HNC}(J=1-0)$, and $\operatorname{HN}^{13} \mathrm{C}(J=1-0)$, the frequencies of which lie in the range $87-110 \mathrm{GHz}$. These lines were observed in the frequency switching mode with frequency throws of 9 or $15 \mathrm{MHz}\left({ }^{13} \mathrm{CO}\right)$. The spectra were calibrated by the chopper-wheel method. The total system temperature was usually about $400 \mathrm{~K}$. The spectral figures and maps included in this paper are presented on the $T_{\mathrm{A}}^{*}$ scale. In the calculation of column densities these intensities were converted to the radiation temperatures, $T_{\mathrm{R}}$, by dividing them by the assumed source-beam coupling efficiency. This value is chosen midway the mainbeam efficiency (0.74) and the moon efficiency (0.9) of the telescope at $110 \mathrm{GHz}$, i.e. $\eta=0.8$.

Cha III-A and Cha III-B are two cold dust column density peaks selected from the IRAS difference $I(100 \mu \mathrm{m})-5 I(60 \mu \mathrm{m})$ map. The following nominal centres were used in the mappings (equinox 1950.0): $\alpha=$ $12^{\mathrm{h}} 51^{\mathrm{m}} 04^{\mathrm{s}}, \delta=-78^{\circ} 37^{\prime} 33^{\prime \prime}$ (Cha III-A); $\alpha=12^{\mathrm{h}} 49^{\mathrm{m}} 01^{\mathrm{s}}$, $\delta=-79^{\circ} 27^{\prime} 55^{\prime \prime}$ (Cha III-B). The spacing was usually $1^{\prime}$, but in the brightest regions $30^{\prime \prime}$ was used. These objects were mapped mainly in $\mathrm{C}^{18} \mathrm{O}$ but also at selected positions in the $(J=1-0)$ transitions of $\mathrm{C}^{17} \mathrm{O}, \mathrm{HCN}, \mathrm{HNC}$ and $\mathrm{HN}^{13} \mathrm{C}$.

The Serpentine was mapped in ${ }^{13} \mathrm{CO}$ and $\mathrm{C}^{18} \mathrm{O}$. The spacing used in these $\mathrm{C}^{18} \mathrm{O}$ maps was $40^{\prime \prime}$, whereas for ${ }^{13} \mathrm{CO}$ we used $80^{\prime \prime}$. The integration times were $60 \mathrm{~s}$ and $30 \mathrm{~s}$ for $\mathrm{C}^{18} \mathrm{O}$ and ${ }^{13} \mathrm{CO}$, respectively. The ${ }^{13} \mathrm{CO}$ and $\mathrm{C}^{18} \mathrm{O}$ observations cover the same areas except for Cha III$\mathrm{C}$, where no ${ }^{13} \mathrm{CO}$ data were collected. Moreover, region Cha III-E emits only very weak ${ }^{13} \mathrm{CO}$ emission, and was not observed for $\mathrm{C}^{18} \mathrm{O}$. A nominal map centre was selected at: $\alpha=12^{\mathrm{h}} 21^{\mathrm{m}} 50^{\mathrm{s}}, \delta=-80^{\circ} 17^{\prime} 20^{\prime \prime}(1950.0)$.

\section{Results}

\subsection{Line profiles}

The spectra obtained towards two selected locations, one in Cha III-A and one in Cha III-B are shown in Fig. 3. All the observed lines except $\mathrm{C}^{18} \mathrm{O}(J=1-0)$ have hyperfine components, and these are indicated in the figure. The $\mathrm{C}^{18} \mathrm{O}$ and $\mathrm{C}^{17} \mathrm{O}$ lines are about 25 percent weaker towards Cha III-B, which implies that the total gas column density is slightly smaller towards the latter source. The difference between the two locations is more significant in 

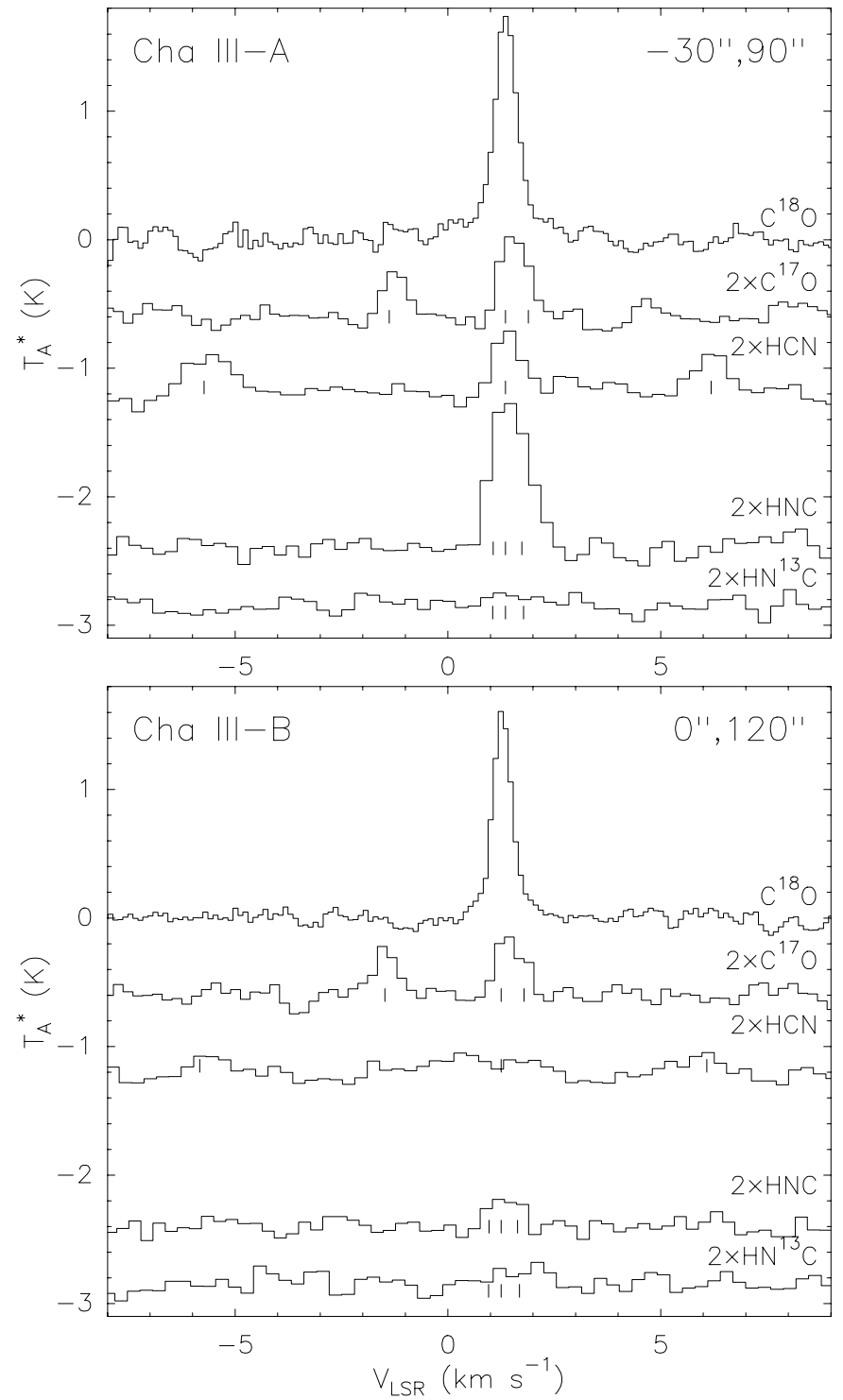

Fig. 3. The observed spectra towards one location in Cha IIIA (upper panel) and one in Cha III-B (lower panel). The four weaker lines are multiplied by 2 . All these have three hyperfine components due to the nuclear spins of ${ }^{17} \mathrm{O}(I=5 / 2)$ and ${ }^{14} \mathrm{~N}$ $(I=1)$, and their locations are indicated with ticks below the spectra. For $\mathrm{C}^{17} \mathrm{O}(J=1-0)$ these components are (from left to right) $F=5 / 2 \rightarrow 5 / 2, F=7 / 2 \rightarrow 5 / 2$ and $F=3 / 2 \rightarrow 5 / 2$; for $\operatorname{HCN}(J=1-0)$ they are $F=0 \rightarrow 1, F=2 \rightarrow 1$, and $F=1 \rightarrow 1$; and for $\operatorname{HNC}(J=1-0)$ and $\operatorname{HN}^{13} \mathrm{C}(J=1-0)$ they are $F=1 \rightarrow 1, F=2 \rightarrow 1$, and $F=0 \rightarrow 1$.

the intensities of $\mathrm{HCN}$ and $\mathrm{HNC}$. Both isomers are clearly detected towards Cha III-A, whereas Cha III-B shows only weak emission from $\mathrm{HNC}$. $\mathrm{HN}^{13} \mathrm{C}$ was not detected towards either of the sources. HCN and HNC have been observed to be nearly equally abundant in dark clouds (e.g. Talbi \& Herbst 1998), but the lines of HNC are easier to detect because the hyperfine splitting is smaller than for HCN. It is not conceivable that the fractional abundances of HCN and HNC would be very different in Cha III-A and Cha III-B. Therefore their column densities are probably similar towards both objects, and the fact that the lines

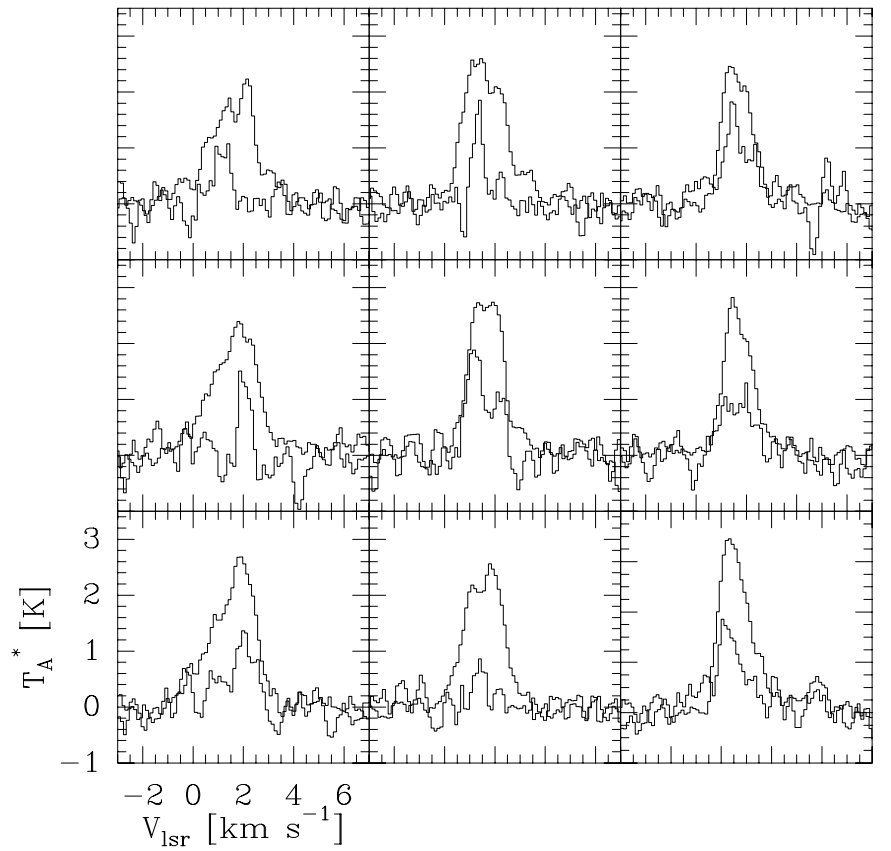

Fig. 4. Examples of line profiles of ${ }^{13} \mathrm{CO}$ and $\mathrm{C}^{18} \mathrm{O}$ (intensity multiplied by factor of 3) collected over the region in the northeastern part of Cha III-D (the tilted filament; central position $\alpha=12^{\mathrm{h}} 40^{\mathrm{m}}, \delta=-79^{\circ} 00^{\prime}$; grid step size $\left.80^{\prime \prime}\right)$. In this area the profiles are double peaked.

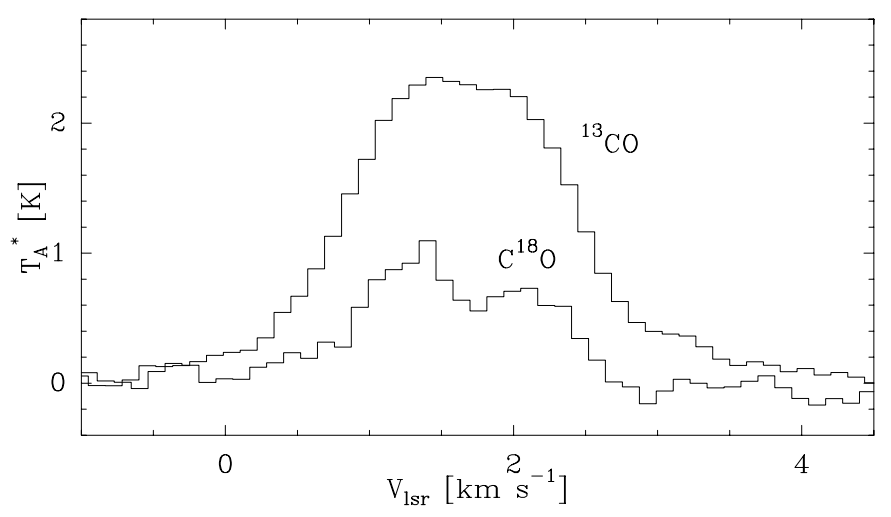

Fig. 5. Comparison between the averaged ${ }^{13} \mathrm{CO}$ and $\mathrm{C}^{18} \mathrm{O}$ line profiles over the 9 positions presented in Fig. 4.

were very weak in Cha III-B must be due to a lower average density there. HCN and HNC have large permanent dipole moments and their first rotational levels require a density of $10^{5} \mathrm{~cm}^{-3}$ to be excited at typical dark cloud temperatures.

Figure 4 gives examples of ${ }^{13} \mathrm{CO}$ and $\mathrm{C}^{18} \mathrm{O}$ profiles collected over an area in the upper, eastern part of the tilted filament Cha III-D. The grid step size is $80^{\prime \prime}$. The ${ }^{13} \mathrm{CO}$ profiles are double-peaked in this area. The $\mathrm{C}^{18} \mathrm{O}$ lines (multiplied by a factor of 3 in Fig. 4) are rather weak in certain directions. The large variation in relative intensities of the two lines reflects primarily noise in the $\mathrm{C}^{18} \mathrm{O}$ spectra. By integrating the emission over several positions, more reliable intensity ratios between the two isotopes can be derived. Figure 5 shows such an example, where both 


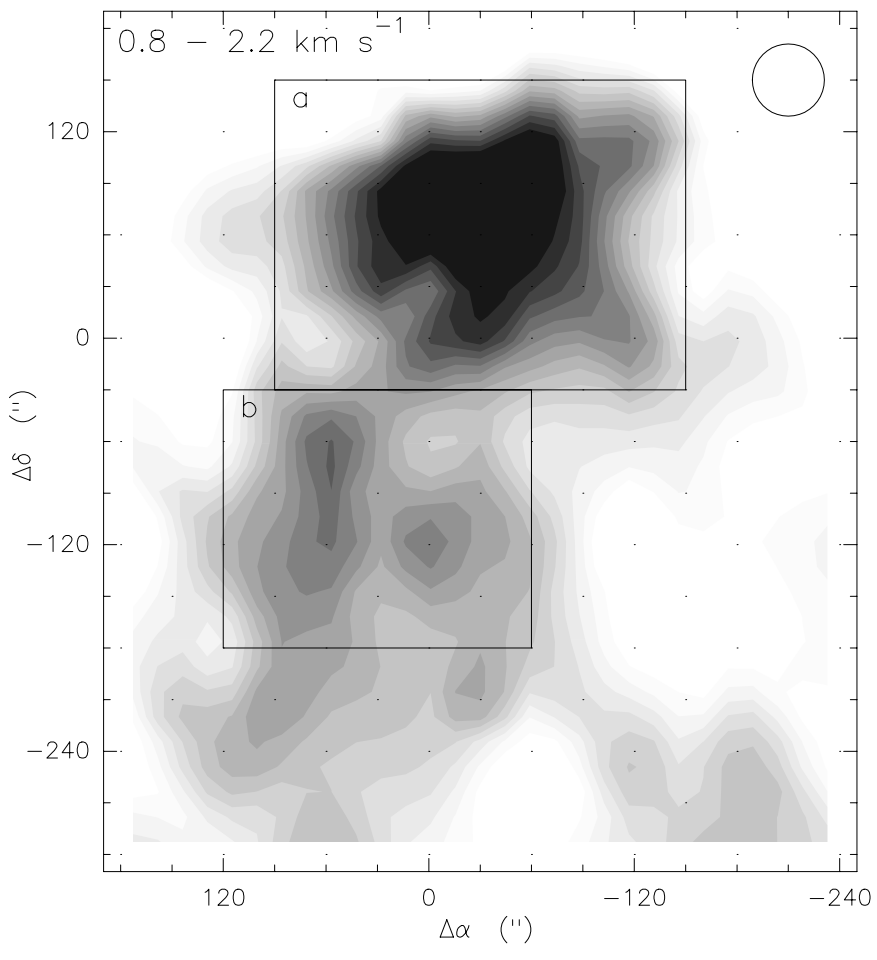

Fig. 6. The $\mathrm{C}^{18} \mathrm{O}(J=1-0)$ integrated intensity map of region Cha III-A. The lowest grey scale level corresponds to $0.1 \mathrm{~K} \mathrm{~km} \mathrm{~s}^{-1}$. Levels increase in steps of $0.1 \mathrm{~K} \mathrm{~km} \mathrm{~s}^{-1}$. The beam size is indicated in the top right. Two identified clumps are labeled in boxes. The map centre is at $\mathrm{RA}=12^{\mathrm{h}} 51^{\mathrm{m}} 04^{\mathrm{s}}$, Dec $=-78^{\circ} 37^{\prime} 33^{\prime \prime}(1950.0)$.

profiles were integrated over the 9 positions presented in Fig. 4. One can see that also the averaged $\mathrm{C}^{18} \mathrm{O}$ spectrum over this region has a double-peaked profile.

\subsection{The clumps in Cha III}

The $\mathrm{C}^{18} \mathrm{O}(J=1-0)$ integrated intensity maps of the regions Cha III-A and Cha III-B are shown in Figs. 6 and 7 . In both regions, the $\mathrm{C}^{18} \mathrm{O}$ emission arises in a narrow velocity range between 0.8 and $2.2 \mathrm{~km} \mathrm{~s}^{-1}$. Cha III-A, which is located near the tip of one of the branches of the north-south oriented filament, contains only one prominent, roughly spherical clump, labeled Cha III-A: $a$, and another, small condensation on its southern side (Cha III$\mathrm{A}: b)$. The region Cha III-B is fragmented into a number of small clumps, clustering in its southern part. We indentified altogether 19 clumps, and labelled them Cha III $\mathrm{B}: a$ to $s$. The most prominent individual condensation in Cha III-B is the elongated clump designated " $n$ ".

In the Serpentine a clump is defined as any collection of at least two adjacent raster positions which satisfy the condition $\int T_{\mathrm{A}}^{*} \mathrm{~d} v \geq 0.5 \mathrm{~K} \mathrm{~km} \mathrm{~s}^{-1}$, and which form a separate entity. In this way we find altogether 17 clumps, and designate them Serpentine: $a$ to $q$. They are shown in contours in Fig. 8 where the IRAS cold dust map, $I(100 \mu \mathrm{m})-5 \times I(60 \mu \mathrm{m})$ forms the background. The $\mathrm{C}^{18} \mathrm{O}$ clumps are confined along the Serpentine, and areas of

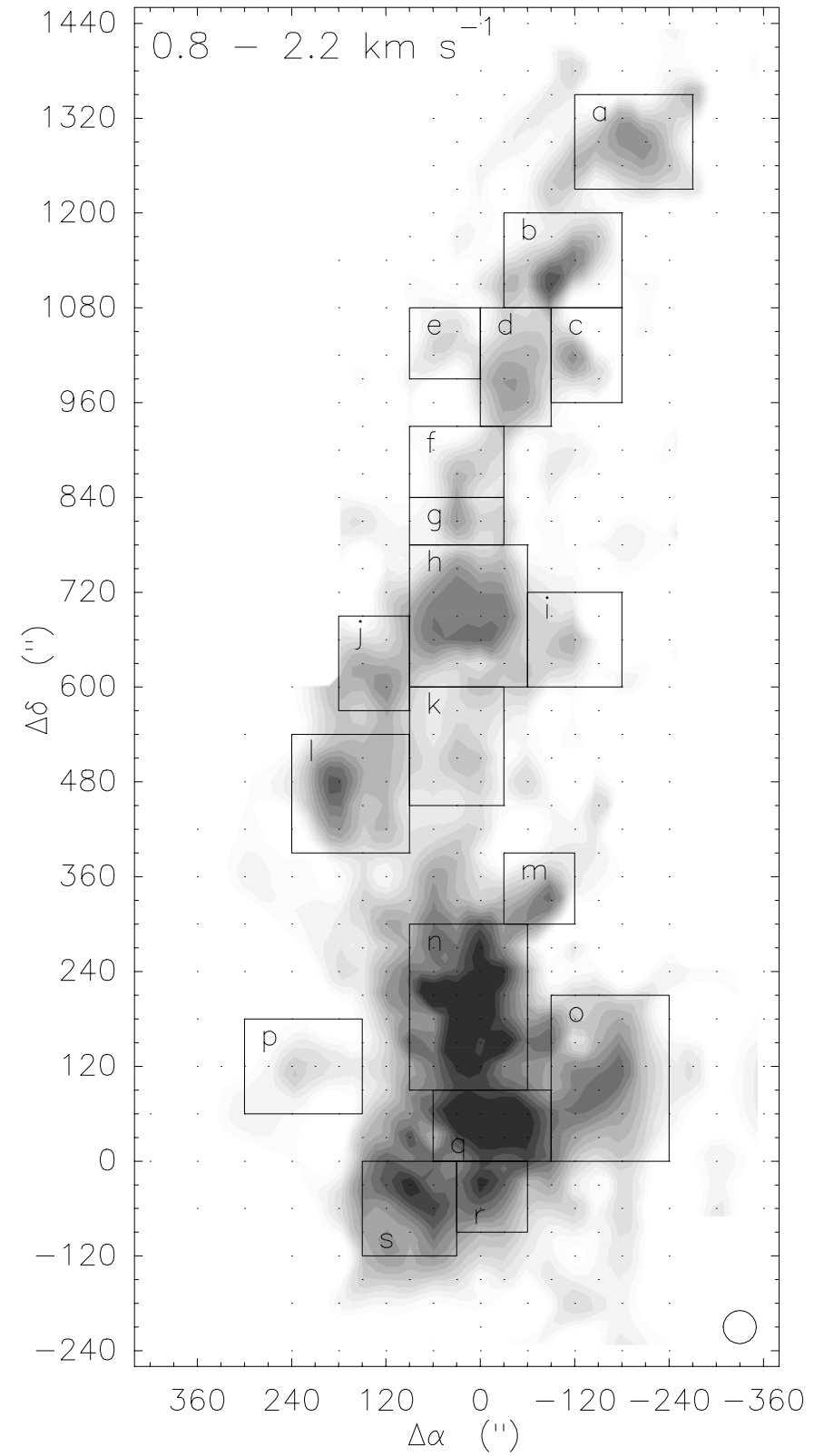

Fig. 7. The $\mathrm{C}^{18} \mathrm{O}(J=1-0)$ integrated intensity map of region Cha III-B (intensity scale as in Fig. 6). Nineteen clumps are identified and labeled in boxes. The map centre is at RA = $12^{\mathrm{h}} 49^{\mathrm{m}} 01^{\mathrm{s}}$, Dec $=-79^{\circ} 27^{\prime} 55^{\prime \prime}(1950.0)$.

enhanced $\mathrm{C}^{18} \mathrm{O}$ emission coincide well with areas of pronounced cold dust emission.

The masses of the clumps and their dynamical states are estimated with the aid of the integrated intensities and widths of the $\mathrm{C}^{18} \mathrm{O}(J=1-0)$ lines. Firstly, we have derived the $\mathrm{C}^{18} \mathrm{O}$ column densities, $N\left(\mathrm{C}^{18} \mathrm{O}\right)$, from the integrated intensities by assuming optically thin emission and LTE with the excitation temperature, $T_{\mathrm{ex}}=10 \mathrm{~K}$. The $\mathrm{C}^{18} \mathrm{O}$ column densities have been converted to the total gas column densities by assuming that $N\left(\mathrm{C}^{18} \mathrm{O}\right) / N\left(\mathrm{H}_{2}\right)=$ $2 \times 10^{-7}$, and that the gas consists of $\mathrm{H}_{2}$ molecules and He atoms with percentages of $80 \%$ and $20 \%$, respectively. Accordingly, the mean molecular mass has been assumed 


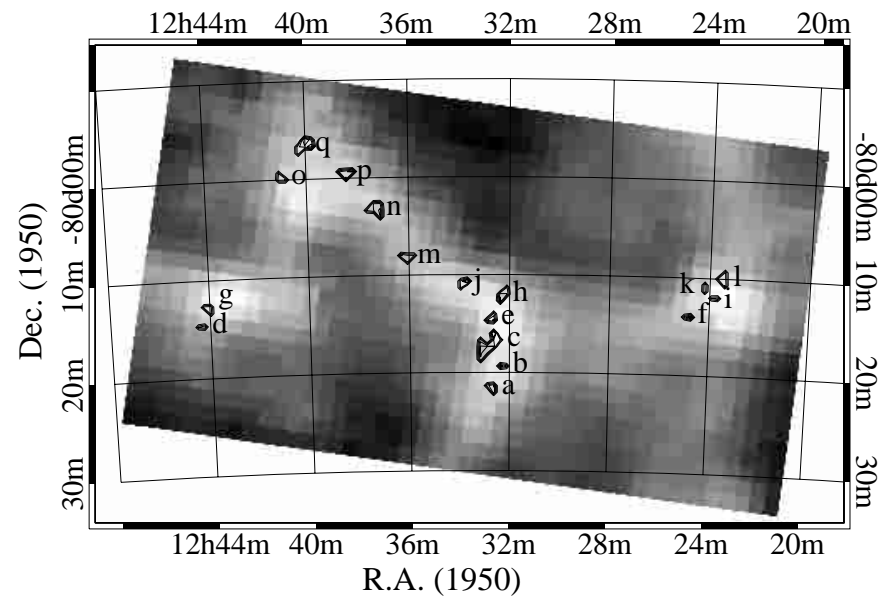

Fig. 8. Contours of the most intense $\mathrm{C}^{18} \mathrm{O}$ clumps (labeled) in the Serpentine. The IRAS cold dust map forms the background.

to be 2.4 a.m.u. The assumed $\mathrm{C}^{18} \mathrm{O} / \mathrm{H}_{2}$ column density ratio is in accordance with the results of Frerking et al. (1982) for dense cores in Taurus, and of Harjunpää \& Mattila (1996) for the Cha I and R CrA complexes, when using the value of the $N\left(\mathrm{H}+\mathrm{H}_{2}\right) / E(B-V)$ ratio derived by Bohlin et al. (1978). One should note, however, that in the case of Cha III it could be justified to adopt the value $110^{-7}$ for $N\left(\mathrm{C}^{18} \mathrm{O}\right) / N\left(\mathrm{H}_{2}\right)$ derived by Harjunpää $\&$ Mattila (1996) for the Coalsack, which is another nonstarforming cloud. In the latter case the masses and ratios of potential to kinetic energies derived here would increase by a factor of two.

The clump mass has been calculated by summing the gas column densities towards each pixel in the clump region and multiplying this sum by the physical area of one pixel. The latter has been calculated using the adopted distance of $150 \mathrm{pc}$ to the cloud. The clump radii have been derived from geometrical averages of the half-power radii, and these have been used together with the average linewidths within the clumps to derive the ratios of the potential to the kinetic energies. In the latter derivation each clump has been approximated by a homogeneous sphere.

The results of these calculations are presented in Table 1. The columns of this table are: (1) the clump designation (indicated in Figs. 6-8); (2) mass; (3) radius; (4) velocity in local standard of rest of individual clumps; (5) average $\mathrm{C}^{18} \mathrm{O}$ linewidth at half intensity, and corrected for instrumental broadening; (6) ratio of the potential to kinetic energy; (7) and (8) coordinates of the centre of mass with respect to the map centre. The estimated errors in the parameters in Cols. (4) and (5) are given in parentheses. Also given is the total mass as derived from the detected $\mathrm{C}^{18} \mathrm{O}$ emission over each region.

Combining the information for all areas we find that the ratio of the potential to kinetic energy is small, and below the virial value, for all clumps. The possible overestimation of the fractional $\mathrm{C}^{18} \mathrm{O}$ abundance by a factor of two (see above) would not change this fact. The clumps are of small mass, less than $1 M_{\odot}$ with only two excep- tions, namely Cha III-A: $a$ and Cha III-B: $n$ with masses of 1.5 and $1.4 M_{\odot}$, respectively. The clump mass distribution shows a decrease with mass in line with what has been found for other regions. However, most of the clumps are of very small mass. The line widths measured for the clumps are similar for all regions. The ${ }^{13} \mathrm{CO}$ emission is smoother, less clumpy, and shows that the denser clumps are embedded in an interclump medium of low density. Even for area Cha III-E, where the extinction is low, ${ }^{13} \mathrm{CO}$ was detected. The corresponding profiles, showing only one component, are symmetric and change little in intensity over the area.

Finally, we also compared our clump parameters by using the different procedure developed by Williams et al. (1994). Although the two programs agree on most of the clumps listed in Table 1, there are some differences regarding crowded areas, especially the southern part of Cha III-B. Moreover, the Williams et al. program gives smaller velocity dispersions for the clumps because these are calculated using only those channels where the intensity exceeds the given starting level. Our estimates are based on Gaussian fits to the full averaged $\mathrm{C}^{18} \mathrm{O}$ spectra within each clump. We think this is appropriate in a thin, filamentary cloud with typically only one radial velocity component in each line of sight. The differences are, however, of no importance to the subsequent discussion of the physics of the clumpy filaments.

\subsection{Velocity components and gradients}

From Table 1 we can conclude that Cha III clumps have an average velocity of $1.4 \mathrm{~km} \mathrm{~s}^{-1}$ with a dispersion of only $0.2 \mathrm{~km} \mathrm{~s}^{-1}$. We have made a number of cuts, showing the ${ }^{13} \mathrm{CO}$ and $\mathrm{C}^{18} \mathrm{O}$ line profiles across and along the various filaments at different positions. The most pronounced gradient in velocity was found in Cha III-B, in directions east-west, but the maximum shift in velocity amounts only to about $0.5 \mathrm{~km} \mathrm{~s}^{-1}$. Our general finding is that only very small velocity gradients are present in Cha III.

However, the ${ }^{13} \mathrm{CO}$ profiles have double peaks or asymmetric shapes over certain areas in the Serpentine. The $\mathrm{C}^{18} \mathrm{O}$ profile in Fig. 5 shows that these components are real velocity components, and not due to self reversals. The separation of the components is small, less than $1 \mathrm{~km} \mathrm{~s}^{-1}$ as a rule. In order to overview the velocity pattern, we have used Positive Matrix Factorization (PMF) to separate two velocity components. PMF is a factor analytical method that describes observations as the product of two matrices, one defining the basic spectral shapes and the other giving the intensity of these basic components in each of the observed spectra (Juvela et al. 1996). $\mathrm{PMF}$ is in this respect similar to e.g. the principal component analysis, PCA (Murtagh \& Heck 1994) but with two important differences. Firstly, in PMF the elements of the factor matrices are required to be non-negative, and this is often enough to produce a unique and physically meaningful solution. In PCA the computed spectral components will have many negative values, and the correspondence 
Table 1. Characteristics of clumps in Chamaeleon III.

Cha III-A, total mass $5 M_{\odot}$

\begin{tabular}{ccccccrr}
\hline Clump & Mass & Radius & $\left\langle V_{\text {LSR }}>\right.$ & $\langle\Delta v>$ & $\left|E_{\text {pot }}\right| / E_{\text {kin }}$ & \multicolumn{2}{c}{ C.M. } \\
& & & & & & $\begin{array}{r}\Delta \alpha \\
\left({ }^{\prime \prime}\right)\end{array}$ & $\begin{array}{r}\Delta \delta \\
\left({ }^{\prime \prime}\right)\end{array}$ \\
\hline $\mathrm{a}$ & $\left(M_{\odot}\right)$ & $(\mathrm{pc})$ & $\left(\mathrm{km} \mathrm{s}^{-1}\right)$ & $\left(\mathrm{km} \mathrm{s}^{-1}\right)$ & & -33 & 56 \\
$\mathrm{~b}$ & 0.6 & 0.07 & $1.41(0.01)$ & $0.67(0.01)$ & 0.33 & 43 & -101 \\
\hline
\end{tabular}

Cha III-B, total mass $21 M_{\odot}$

\begin{tabular}{ccccccrr}
\hline Clump & Mass & Radius & $\left\langle V_{\text {LSR }}\right\rangle$ & $\langle\Delta v\rangle$ & $\left|E_{\text {pot }}\right| / E_{\text {kin }}$ & $\begin{array}{r}\text { C.M. } \\
\Delta \alpha \\
\left({ }^{\prime \prime}\right)\end{array}$ & $\begin{array}{r}\Delta \delta \\
\left({ }^{\prime \prime}\right)\end{array}$ \\
\hline $\mathrm{a}$ & $\left(M_{\odot}\right)$ & $(\mathrm{pc})$ & $\left(\mathrm{km} \mathrm{s}^{-1}\right)$ & $\left(\mathrm{km} \mathrm{s}^{-1}\right)$ & & -189 & 1284 \\
$\mathrm{~b}$ & 0.5 & 0.05 & $1.64(0.05)$ & $1.26(0.09)$ & 0.06 & -90 & 1123 \\
$\mathrm{c}$ & 0.4 & 0.04 & $1.26(0.07)$ & $1.05(0.12)$ & 0.07 & -110 & 1025 \\
$\mathrm{~d}$ & 0.5 & 0.03 & $1.43(0.05)$ & $0.58(0.09)$ & 0.11 & -45 & 1002 \\
$\mathrm{e}$ & 0.2 & 0.05 & $1.44(0.02)$ & $0.55(0.03)$ & 0.20 & 35 & 1030 \\
$\mathrm{f}$ & 0.1 & 0.03 & $1.46(0.02)$ & $0.54(0.06)$ & 0.11 & 7 & 882 \\
$\mathrm{~g}$ & 0.1 & 0.02 & $1.34(0.01)$ & $0.63(0.03)$ & 0.08 & 11 & 806 \\
$\mathrm{~h}$ & 0.7 & 0.05 & $1.36(0.03)$ & $1.13(0.08)$ & 0.09 & 23 & 693 \\
$\mathrm{i}$ & 0.2 & 0.03 & $1.31(0.05)$ & $1.18(0.10)$ & 0.04 & -83 & 653 \\
$\mathrm{j}$ & 0.4 & 0.04 & $1.27(0.02)$ & $0.74(0.04)$ & 0.12 & 128 & 617 \\
$\mathrm{k}$ & 0.3 & 0.04 & $1.08(0.02)$ & $0.69(0.05)$ & 0.10 & 30 & 529 \\
$\mathrm{l}$ & 0.7 & 0.06 & $1.34(0.02)$ & $0.76(0.05)$ & 0.16 & 154 & 475 \\
$\mathrm{~m}$ & 0.2 & 0.03 & $1.41(0.03)$ & $0.78(0.07)$ & 0.09 & -69 & 328 \\
$\mathrm{n}$ & 1.4 & 0.06 & $1.38(0.01)$ & $0.66(0.02)$ & 0.34 & 14 & 197 \\
$\mathrm{o}$ & 0.8 & 0.06 & $1.77(0.05)$ & $0.95(0.13)$ & 0.13 & -137 & 96 \\
$\mathrm{p}$ & 0.2 & 0.03 & $1.61(0.05)$ & $0.56(0.18)$ & 0.10 & 213 & 117 \\
$\mathrm{q}$ & 0.9 & 0.05 & $1.35(0.01)$ & $0.91(0.03)$ & 0.17 & -16 & 49 \\
$\mathrm{r}$ & 0.4 & 0.04 & $1.16(0.02)$ & $0.83(0.06)$ & 0.13 & -7 & -29 \\
$\mathrm{~s}$ & 0.7 & 0.05 & $1.35(0.02)$ & $1.01(0.05)$ & 0.12 & 79 & -45 \\
\hline
\end{tabular}

The Serpentine, total mass $30 M_{\odot}$

\begin{tabular}{|c|c|c|c|c|c|c|c|}
\hline \multirow[t]{2}{*}{ Clump } & \multirow{2}{*}{$\begin{array}{l}\text { Mass } \\
\left(M_{\odot}\right) \\
\end{array}$} & \multirow{2}{*}{$\begin{array}{c}\text { Radius } \\
(\mathrm{pc}) \\
\end{array}$} & \multirow{2}{*}{$\begin{array}{l}<V_{\mathrm{LSR}}> \\
\left(\mathrm{kms}^{-1}\right)\end{array}$} & \multirow{2}{*}{$\begin{array}{l}<\Delta v> \\
\left(\mathrm{km} \mathrm{s}^{-1}\right)\end{array}$} & \multirow[t]{2}{*}{$\left|E_{\text {pot }}\right| / E_{\text {kin }}$} & \multicolumn{2}{|c|}{ C.M. } \\
\hline & & & & & & $\begin{array}{l}\Delta \alpha \\
\left({ }^{\prime \prime}\right)\end{array}$ & $\begin{array}{l}\Delta \delta \\
\left({ }^{\prime \prime}\right)\end{array}$ \\
\hline $\mathrm{a}$ & 0.2 & 0.03 & $1.40(0.03)$ & $0.82(0.08)$ & 0.06 & 1651 & -254 \\
\hline b & 0.1 & 0.02 & $1.40(0.03)$ & $0.82(0.08)$ & 0.05 & 1582 & -119 \\
\hline $\mathrm{c}$ & 0.8 & 0.05 & $1.63(0.03)$ & $0.79(0.08)$ & 0.16 & 1676 & 5 \\
\hline d & 0.1 & 0.02 & $1.05(0.02)$ & $0.58(0.07)$ & 0.07 & 3418 & 160 \\
\hline $\mathrm{e}$ & 0.2 & 0.03 & $1.34(0.03)$ & $0.75(0.08)$ & 0.07 & 1652 & 171 \\
\hline $\mathrm{f}$ & 0.2 & 0.02 & $1.58(0.03)$ & $0.89(0.10)$ & 0.05 & 456 & 199 \\
\hline $\mathrm{g}$ & 0.2 & 0.03 & $1.40(0.03)$ & $0.82(0.08)$ & 0.05 & 3373 & 266 \\
\hline $\mathrm{h}$ & 0.3 & 0.03 & $1.29(0.03)$ & $0.74(0.10)$ & 0.09 & 1582 & 316 \\
\hline $\mathrm{i}$ & 0.1 & 0.02 & $1.52(0.03)$ & $0.84(0.08)$ & 0.04 & 298 & 320 \\
\hline $\mathrm{j}$ & 0.2 & 0.03 & $1.13(0.04)$ & $0.77(0.11)$ & 0.09 & 1821 & 389 \\
\hline $\mathrm{k}$ & 0.1 & 0.02 & $1.64(0.03)$ & $1.00(0.12)$ & 0.03 & 360 & 380 \\
\hline 1 & 0.3 & 0.03 & $1.41(0.03)$ & $0.93(0.07)$ & 0.05 & 249 & 438 \\
\hline $\mathrm{m}$ & 0.3 & 0.03 & $1.18(0.03)$ & $0.94(0.07)$ & 0.05 & 2162 & 550 \\
\hline $\mathrm{n}$ & 0.5 & 0.04 & $1.26(0.02)$ & $0.80(0.06)$ & 0.10 & 2343 & 845 \\
\hline o & 0.2 & 0.03 & $1.97(0.03)$ & $0.83(0.07)$ & 0.05 & 2905 & 1051 \\
\hline $\mathrm{p}$ & 0.3 & 0.03 & $1.48(0.02)$ & $0.83(0.07)$ & 0.08 & 2517 & 1069 \\
\hline q & 0.5 & 0.04 & $1.40(0.03)$ & $0.82(0.08)$ & 0.12 & 2758 & 1249 \\
\hline
\end{tabular}

The parameters for the Serpentine clump $n$ are provisional. 

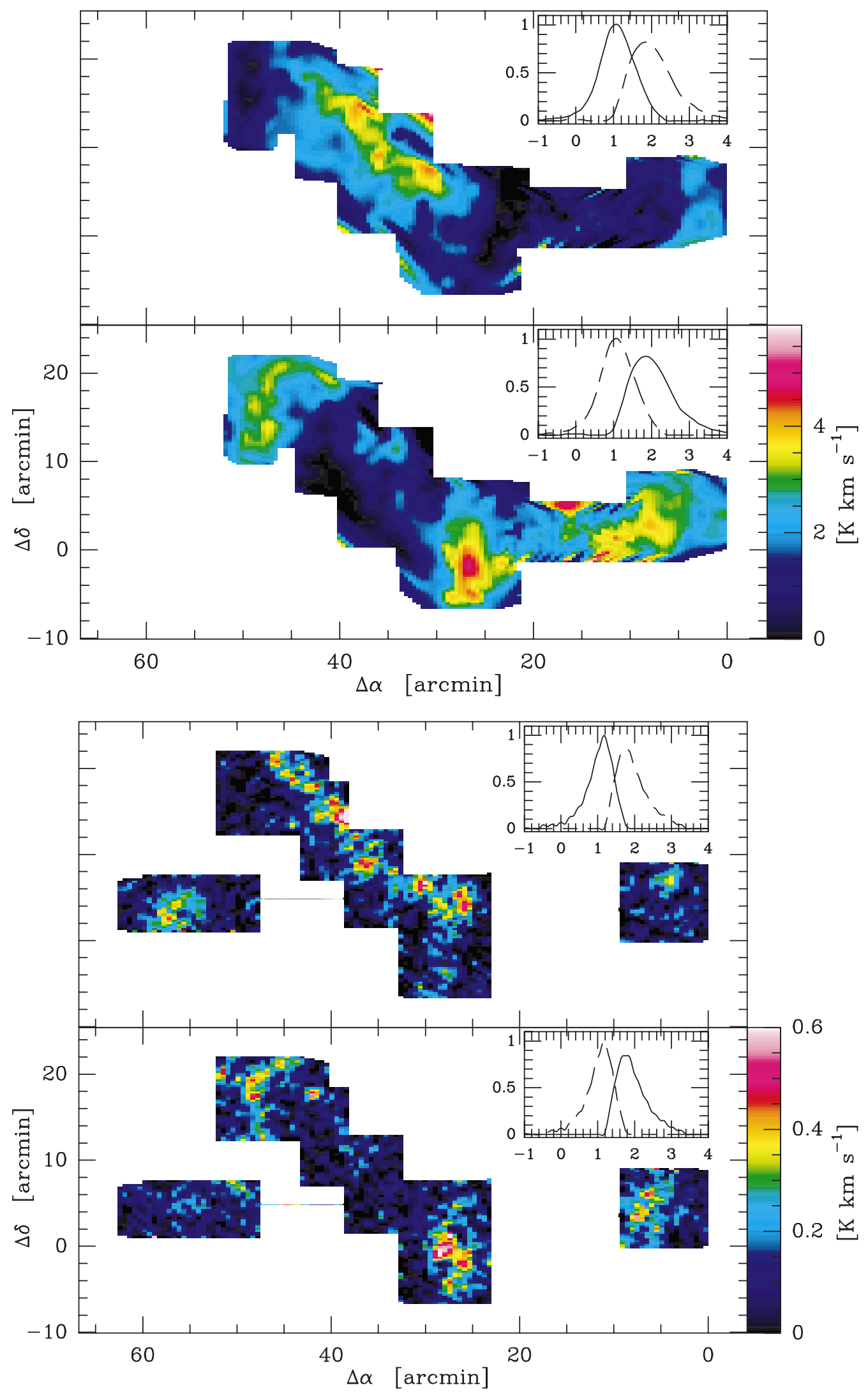

Fig. 9. Certain regions in the Serpentine show two distinct velocity components. The figures present the results of the PMF analysis of the ${ }^{13} \mathrm{CO}$ (upper panel) and $\mathrm{C}^{18} \mathrm{O}$ spectra (lower panel), assuming two velocity components (see the inserted profiles). The top frames show the integrated intensity maps of the blue-shifted component. Notice the pronounced emission along the tilted filament (Cha III-D). The bottom frames represent the red-shifted gas component where the tilted filament is absent. Instead areas at the upper and lower end of the tilted filament are prominent, and also in Cha III-E and F. We identify these two structures with the system of north-south oriented IRAS dust filaments seen in Fig. 2. 


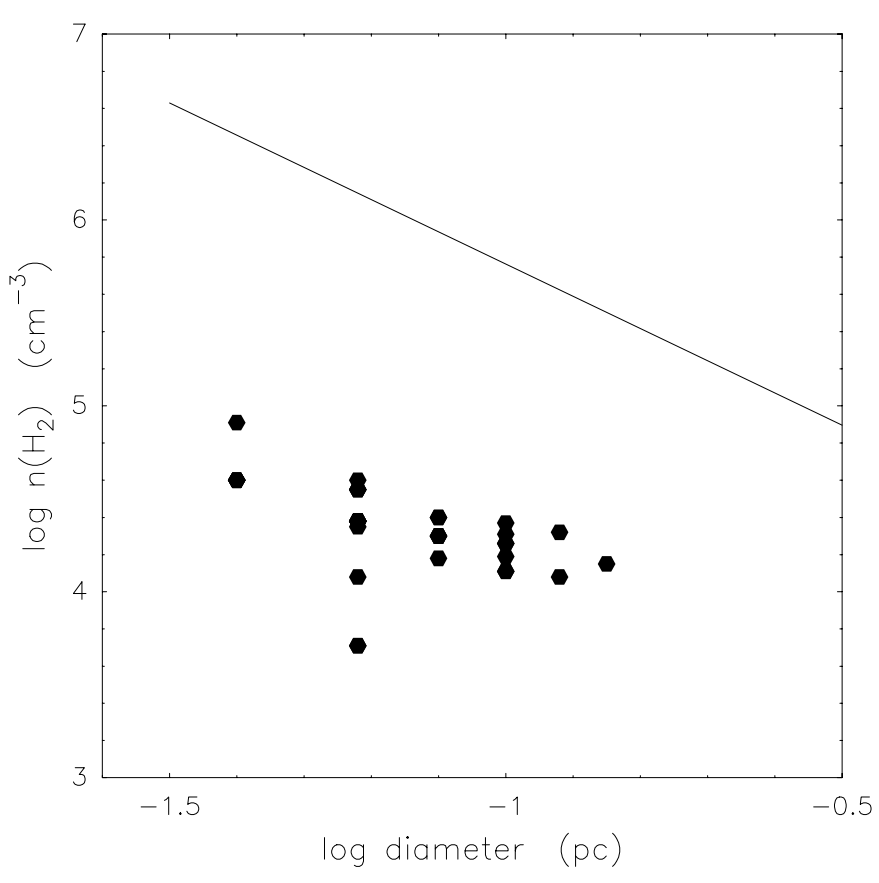

Fig. 10. Density versus diameter for all clumps in Cha III. Some points overlap. For comparison the corresponding relation found for clumps of low mass in other complexes as given by Vallée (2000) is shown as a line.

to the observed emission lines is more indirect. Secondly, PMF makes optimal use of the available error estimates, and the results will therefore not be biased even if the noise level of the spectra varies.

The factorizations were calculated with a program developed by P. Paatero (Paatero 1999). The results of the analysis of the ${ }^{13} \mathrm{CO}$ and $\mathrm{C}^{18} \mathrm{O}$ spectra are shown in Fig. 9. The blue-shifted component is confined to the tilted filament (middle of Cha III-D). The red-shifted component is pronounced in the north-south direction at two areas, namely at the upper and lower part of the tilted filament. These areas are also the locations where the system of thin filaments of warmer dust crosses the tilted filament of colder dust in the north-south direction (see Fig. 2 above and Fig. 4 in Boulanger et al. 1998). Cha III-F and parts of Cha III-E belong to the red-shifted gas component. Hence both the IRAS maps and our CO maps indicate that there are two separate, but interwoven systems of extended filaments in Cha III. The systems have different orientations which give rise to the zig-zag pattern seen on optical images and also to enhanced FIR emission in the line-ofsight to locations where filaments cross.

At the crossings distinct two-component spectra are seen in both $\mathrm{C}^{18} \mathrm{O}$ and ${ }^{13} \mathrm{CO}$. The clump in the Serpentine, designated " $q$ ", is located in one crossing. Both velocity components are present and it is likely that Serpentine: $q$ is composed of two clumps, where the red-shifted clump is shifted to the south-east of the blue-shifted clump.

Hence, there is no evidence of substantial disturbances of the Cha III molecular clouds, nor of rotation of these clumpy filaments. Small shifts, of a few tenths of $\mathrm{kms}^{-1}$, can be traced along certain filaments, on geometrical scales of the order of 1 pc.

\section{Discussion}

The Cha III molecular complex is composed of a network of thin but extended wavy filaments. Only small velocity gradients exist along individual filaments, but different filaments may have slightly different velocities resulting in two velocity components at crossings.

The filaments break up in a number of tiny clumps, "threaded" on the filaments and with masses considerably smaller than $1 M_{\odot}$. The clumps of much larger mass reported by Vilas-Boas et al. (1994), Mizuno et al. (1998, 1999) and Boulanger et al. (1998) are in some cases related to assemblies of clumps discovered in our survey. This increase in granularity with increasing spatial resolution is a rather typical outcome in the studies of molecular clouds, with or without star fomation (see e.g. Langer et al. 1995; Falgarone \& Phillips 1996 and Kramer et al. 1998), and opens the question: are the clumps defined in our survey composed of even smaller clumps. We note that Cha III-B: $n$ shows irregularities hinting at such substructures.

\subsection{Clump properties}

By assuming that our filaments are axi-symmetric we have computed the volumes emitting the bulk of the $\mathrm{CO}$ emission. For the Serpentine we measured an average thickness of 3.3 ( $0.14 \mathrm{pc}$ at the adopted distance). The corresponding volume is computed assuming cylindrical geometry and a correction for the most probable inclination. The Serpentine filaments in Cha III-D, including the tilted filament, then has a total length of $1.85 \mathrm{pc}$. Cha III-B was described as a circular core with a conic extension. Accounting for the difference in sensitivity between the surveys of the Serpentine and of Cha III-B, we can conclude that the number density of clumps is roughly the same over the entire ChaIII complex and the corresponding volume filling factors of clumps are similar, about $7 \%$.

The range of average number densities of individual clumps is $n\left(\mathrm{H}_{2}\right)=1 \times 10^{4}-8 \times 10^{4} \mathrm{~cm}^{-3}$ with about the same distribution in Cha III-B and the Serpentine, and with no obvious correlation with mass. Weak ${ }^{13} \mathrm{CO}$ emission extends far out from the filaments but peaks in regions of enhanced $\mathrm{C}^{18} \mathrm{O}$ emission. The $\mathrm{C}^{18} \mathrm{O}$ column density in the neighbourhood of clumps ChaIII-D: $c, e, h$ and $p$ has an average value of $4 \times 10^{14} \mathrm{~cm}^{-2}$. By assuming that the fractional $\mathrm{C}^{18} \mathrm{O}$ abundance is $2 \times 10^{-7}$, this can be converted to an average molecular hydrogen column density, $N\left(\mathrm{H}_{2}\right)$, of $2 \times 10^{21} \mathrm{~cm}^{-2}$. From this we can derive that that the number density of the interclump medium in Cha III- $D$ is about $4 \times 10^{3} \mathrm{~cm}^{-3}$. Similar values apply for Cha III-B. Hence the density contrast between clump and interclump gas is low, around 10 . 
The maps of the Cha III clumps could therefore be described as a landscape of irregular density enhancements in the volumes of molecular gas. The clumps are very different from those discussed by e.g. Vallée (2000), which is demonstrated in Fig. 10. Most clumps are distinct, however, but especially in the Cha III-B region there are also examples of rather loose structures. Also, the values of $\left|E_{\text {pot }}\right| / E_{\text {kin }}$ for the clumps in Table 1 are very small in comparison to the the clumps investigated by Vallée (2000). The kinetic energies of our clumps are dominated by turbulence, and most clumps are not even close to the critical mass for contraction, if gravity is the only counteracting force.

Both Cha III-B and the Serpentine the clumps are clearly lined up in long structures which well coincide with the IRAS filaments. This is particularly evident in the tilted filament where the clumps are arranged like pearls in a necklace. The velocity differences are moderate and of the order of $0.2 \mathrm{~km} \mathrm{~s}^{-1}$. Nevertheless, with similar velocity dispersion perpendicular to the line-of-sight the observed coherency of the clumps within the filament would be lost on a dynamical timescale of $t_{\mathrm{dyn}} \approx 4 \times 10^{5}$ years.

Collisions between clumps could lead to a merging of clumps, followed by subsequent gravitational collapse. However, the timescale of collisions is $t_{\mathrm{col}} \approx 9 \times 10^{6}$ years much longer than the dynamical timescale. Furthermore, multiple collisions must take place before gravitational collapse is possible.

The fact that most clumps are far from virial equilibrium, and the indication of substructures in those which are, give a hint on why there are no recognized sites of star formation in the complex.

\subsection{Threaded clumps?}

The lining-up of clumps can probably not be considered a coincidence. An important question is therefore what mechanism could protect the clumps from leaving the thin filaments on short timescales? One solution to this problem may be that the clumps do not survive for times longer than $t_{\mathrm{dyn}}$ before they are dissolved. Another possibility is that they live longer, and that there exists a restoring force which is capable of keeping the clumps inside the filament. A natural agent for such a restoring force is the magnetic field. Under the assumption that there exists a magnetic field in the filament that is frozen in to the clumps, they will be subject to magnetic tension representing a restoring force. Each of the clumps may oscillate back and forth perpendicular to the filament, similar to a mass load on an elastic string.

In order to find out the strength of the magnetic field that is required to keep the oscillating clumps within the filament, we first consider a very simple model where a magnetic field, $B_{\mathrm{c}}$, runs along the filament and penetrates the clumps. The clumps are supposed to be equidistant along the filament with the separation $l_{\mathrm{c}}$, and to oscillate perpendicular to the filament as a standing wave with

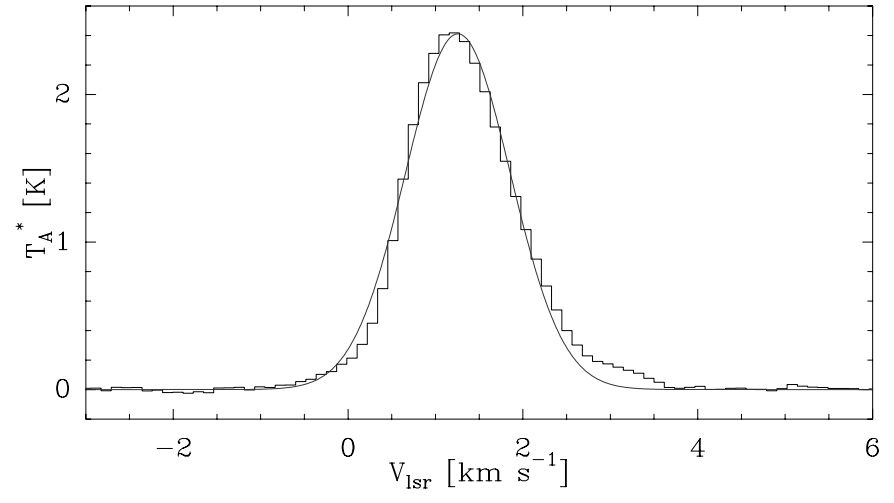

Fig. 11. This ${ }^{13} \mathrm{CO}$ spectrum is a mean of several spectra collected outside the clumps in Cha III-D. The profile is well accounted for by a single Gaussian profile. There is no evidence for wings related to high velocity turbulence.

a wavelength equal to $2 l_{\mathrm{c}}$ (a standing Alfvén wave in a medium of discrete mass distribution). The radius, mass and maximum velocity of the clumps are $R_{\mathrm{c}}, M_{\mathrm{c}}$ and $v_{\mathrm{c}}$, respectively. If the amplitude of the oscillation is not to exceed the radius of the filament, $R_{\mathrm{f}}$, the magnetic field has to fulfill the requirement

$$
B_{\mathrm{c}} \geq B_{0} \equiv\left\{v_{\mathrm{c}} /\left(R_{\mathrm{c}} R_{\mathrm{f}}\right)\right\}\left(M_{\mathrm{c}} l_{\mathrm{c}}\right)^{1 / 2} .
$$

Choosing parameter values valid for the tilted filament, $l_{\mathrm{c}}=0.2 \mathrm{pc}, R_{\mathrm{c}}=0.03 \mathrm{pc}, M_{\mathrm{c}}=0.3 M_{\odot}, v_{\mathrm{c}}=200 \mathrm{~m} \mathrm{~s}^{-1}$, and $R_{\mathrm{f}}=0.07 \mathrm{pc}$, we find that the minimum magnetic field needed is $B_{0}=19 \mu \mathrm{G}$. This minimum field seems to be well below the magnetic field expected in the filament, $\geq 100 \mu \mathrm{G}$ (see Vallée 2000, and references therein). For the wavelength assumed above, the clumps should therefore, in principle, oscillate inside the filament. If the wavelength is larger than the value considered, a proportionally stronger magnetic field is required.

For the filament to survive for reasonable times the internal radial forces must be in equilibrium. Such an equilibrium may exist for several reasons. In the simple example considered above the internal magnetic pressure of the filament may be balanced partly by an outer pressure induced by warm intercloud plasma, and also as pointed out in Sect. 2, there are powerful gas flows in the environment which may interact with the molecular clouds. Another possibility, perhaps even more important, is that also an axially directed electric current flows in the filament giving rise to pinching forces. The magnetic field then turns out to be of helical geometry. A discussion of this matter has been given by Carlqvist (1988).

\subsection{Formation and maintenence of the clumps}

How do the clumps form? As is clear from Table 1 gravity can not keep the clumps together. Using the mean value of the corrected line-width, $0.82 \mathrm{~km} \mathrm{~s}^{-1}$ (The Serpentine, Col. 5, Table 1), and a typical density in the clumps of $4 \times 10^{4} \mathrm{~cm}^{-3}$, we find that the pressure of the clumps is $\sim 2 \times 10^{-10}$ dyn $\mathrm{cm}^{-2}$. Without any collecting force 
the clumps would be dissolved in a typical time of $t_{\mathrm{dis}}=$ $6 \times 10^{4}$ years.

One may ask whether there exists any other force that is capable of keeping the clumps in equilibrium. One possibility might be the pressure force of the interclump medium. Since the ratio of the densities of the clump and interclump media is about 10 , the line-width of the interclump medium has to be about $\sqrt{10} 0.82 \mathrm{~km} \mathrm{~s}^{-1}$ $\sim 2.6 \mathrm{~km} \mathrm{~s}^{-1}$ for an equilibrium to exist. Figure 11 shows the integrated ${ }^{13} \mathrm{CO}$ profile related to the interclump gas in the tilted filament. There is no evidence for any significant high-velocity wings, and we conclude that the pressure force from the interclump medium is insufficient to confine the clumps.

The only reasonable force remaining is the electromagnetic force. To balance the pressure in the clump by means of the magnetic pressure $\mathrm{B}^{2} / 8 \pi$ an external magnetic field of about $60 \mu \mathrm{G}$ is required, provided the field inside the clump is much smaller. This is not an unrealistically large field. It should be stressed that the magnetic field has to be larger outside the clump than inside, so that it encloses the clump in some kind of magnetic bottle.

Another, quite different, possibility is that the clumps are not at all in equilibrium but develop dynamically. If so, clumps may be formed and dissolved successively in relatively short periods. One conceivable mechanism of formation is the sausage-instability which is well-known from fusion physics. In a current carrying filament the sausageinstability may chop the filament into fairly regular pieces resembling the approximately equidistant clumps in the tilted filament. For the sausage-instability to develop an axially directed current is required producing a toroidal magnetic field component, at the same time as the axial magnetic field component can not be too large.

Finally, there is the question of how these thin filaments form and keep their identity over length-scales of even more than a parsec in some cases. The formation of the Chamaeleon complex could be related to global activity in this part of the galaxy, as noted in the Introduction. For the confinement of the long filaments over longer timescales, most likely electromagnetic forces dominate the physics of these objects as discussed in Sect. 5.2.

\section{Conclusions}

Chamaeleon III is a juvenile cloud complex without any obvious signposts of star formation. Our survey of primarily ${ }^{13} \mathrm{CO}$ and $\mathrm{C}^{18} \mathrm{O}$ emission from the area shows that the molecular emission closely follows the distribution of dust emission. The complex contains a number of thin, extended filaments. Individual filaments are wavy, and even sinusoidal. We have shown that:

1. There are at least two systems of filaments, separated in radial velocity and in some regions also by different infrared colours. The systems cross at certain locations, and as a result a very complicated pattern of filaments is seen in projection on the plane-of-sky. For instance the zig-zag shaped features in the southern part of Cha III (the Serpentine) is the result of such an overlap.

2. We identify close to 40 molecular clumps in the filaments. The clumps are all tiny, and the smallest have masses of $0.1-0.3 M_{\odot}$. Only two clumps in the northern part of ChaIII are more massive than $1 M_{\odot}$. Previously recognized clumps of larger mass turn out to be composed of assemblies of smaller clumps, when viewed at the higher spatial resolution used in the present mapping. The volume filling factor of the clumps is about $7 \%$ of the estimated volume for the clouds mapped. The total clump mass amounts to a few tenths of the total mass of the volume.

3. These tiny clumps are of relatively low density, $n\left(\mathrm{H}_{2}\right)=1 \times 10^{4}-8 \times 10^{4} \mathrm{~cm}^{-3}$. The density contrast between clump and interclump gas is only about 10 . The clumps are therefore very different from typical clumps identified in other interstellar clouds.

4. Most clumps have unusually small ratios of the potential to the kinetic energy (mainly turbulent), much below the virial equilibrium values. There is no evidence of any high velocity turbulent motions in the interclump medium that could provide sufficient outer pressure to confine the gravitionally unbound clumps.

5. We measure only small velocity gradients along the filaments. The velocity differences between individual clumps amounts to about $0.2 \mathrm{~km} \mathrm{~s}^{-1}$. Without any restoring force the clumps will leave the filaments on short timescales, $t_{\mathrm{dyn}} \approx 4 \times 10^{5}$ years, which is too short for larger clumps to form from collisions.

From these findings it appears that star formation occurring from gravitational collapse is unlikely in Cha III. In case the fractional $\mathrm{CO}$ abundance is in reality three or four times lower than assumed here, further studies of the two most massive clumps (Cha III-A:a and Cha III-B:n) seem, however, warranted. Two basic questions are how the clumps can be confined, and how they can stay within the filaments. We have explored the possibility that the concentrations are confined by electromagnetic forces, and show that this may work with reasonable assumptions on the required magnetic field strength. In the Serpentine the clumps are approximately equidistant along the main filament. We explore a model where the clumps are threaded on magnetic ropes along the filamentary axis, and oscillate perpendicularly to this axis as a result of magnetic tension forces.

In conclusion we find possible solutions to both the basic questions risen. The magnetic field strengths required are typical for interstellar cloud conditions. These models can be tested by further observations at higher spatial resolution. If there are no restoring forces one must consider the case that the clumps are the result of density fluctuations in the medium.

Acknowledgements. We thank W. Pfau for communicating his unpublished results concerning the distribution of $\mathrm{H} \alpha$ objects 
in Cha III. The Swedish Natural Science Foundation and the Finnish Academy (grant No. 1011055) supported the project.

\section{References}

Alcalá, J. M., Krautter, J., Schmitt, J. H. M. M., et al. 1995, A\&AS, 114, 109

Alcalá, J. M., Krautter, J., Covino, E., et al. 1997, A\&A, 319, 184

Bally, J. 1989, in ed. B. Reipurth, Proc. ESO Workshop on Low mass star formation and pre-main-sequence objects, 1

Bohlin, R. C., Savage, B. D., \& Drake, J. F. 1978, ApJ, 224, 132

Boulanger, F., Bonfman, L., Dame, T. M., et al. 1998, A\&A, 332,273

Cambrésy, L. 1999, A\&A, 345, 965

Carlqvist, P. 1988, Ap\&SS, 144, 73

Carlqvist, P., \& Gahm, G. F. 1992, IEEE Trans. Plasma Sc., 20,867

Carlqvist, P., Kristen, H., \& Gahm, G. F. 1998, A\&A, 332, L5

Carlqvist, P., Gahm, G. F., \& Kristen, H. 2001, Ap\&SS, in press

Caulet, A. 1997, ST-ECF Newsletter, 24, 1

Cleary, M. N., Heiles, C., \& Haslam, C. G. T. 1979, A\&AS, 36, 95

Corradi, W. J. B., Franco, G. A. P., \& Knude, J. 1997, A\&A, 326,1215

Covino, E., Alcala, J. M., Allain, S., et al. 1997, A\&A, 328, 187

Cox, D. P. 1996, in Proc. 11th Astrophys. Meeting. The interplay between massive star formation, the ISM and galaxy evolution, ed. D. Kunth, B. Guideroni, M. HeydariMalayeri, \& Trinh Xuang Thuan (Édition Frontières, Gifsur-Yvette, France), 233

Crutcher, R. M. 1999, ApJ, 520, 706

Eggen, O. J. 1998, AJ, 116, 1314

Elmegreen, B. G., \& Falgarone, E. 1996, ApJ, 471, 816

Falgarone, E., \& Phillips, T. G. 1996, ApJ, 472, 191

Falgarone, E., Pety, J., \& Phillips, T. G. 2001, ApJ, in press

Feigelson, E. D. 1996, ApJ, 468, 306

Feitzinger, J. V., \& Stüwe 1984, A\&AS, 58, 365

Fiege, J. D., \& Pudritz, R. E. 2000a, MN, 311, 85

Fiege, J. D., \& Pudritz, R. E. 2000b, MN, 311, 105

Franco, G. A. P. 1991, A\&A, 251, 581

Frerking, M. A., Langer, W. D., \& Wilson, R. W. 1982, ApJ, 262,590

Gehman, C. S., Adams, F. C., \& Watkins, R. 1996, ApJ, 472, 673

Gry, C., Boulanger, F., Falgarone, E., et al. 1998, A\&A, 331, 1070
Hanawa, T., Nakamura, F., Matsumoto, T., et al. 1993, ApJ, 404, L83

Harjunpää, P., \& Mattila, K. 1996, A\&A, 305, 920

Hartley, M., Manchester, R. N., Smith, R. M., et al. 1986, A\&AS, 63, 27

Hartmann, D., \& Burton, W. B. 1997, Atlas of galactic neutral hydrogen (Cambridge University press)

Hetem, J. C. G., Sanzovo, G. C., \& Lépine, J. R. D. 1988, A\&AS, 76, 347

Juvela, M., Lehtinen, K., \& Paatero, P. 1996, MNRAS, 28, 616

Kramer, C., Stutzki, J., Röhrig, R., et al. 1998, A\&A, 329, 249

Knude, J., \& Høg, E. 1998, A\&A, 338, 897

Langer, W. D., Velusamy, T., Kuiper, T. B. H., et al. 1995, ApJ, 453, 293

Lazarian, A. 1993, Ap\&SS, 206, 37

Lehtinen, K., Mattila, K., \& Schnur, G. F. O. 1995, A\&A, 295, 487

Mizuno, A., Onishi, T., Yonekura, Y., et al. 1995, ApJ, 445, L161

Mizuno, A., Hayakawa, T., Yamaguchi, N., et al. 1998, ApJ, 507, L83

Mizuno, A., Hayakawa, T., Tachihara, K., et al. 1999, PASJ, 51,859

Murtagh, F., \& Heck, A. 1987, in Multivariate Data Analysis, Astrophys. Space Sci. Lib. (Reidel, Dordrecht), 13

Nagai, T., Inutsuka, S.-I., \& Miyama, S. M. 1998, ApJ, 506, 306

Nakajima, Y., \& Hanawa, T. 1996, ApJ, 467, 321

Nakamura, F., Hanawa, T., \& Nakano, T. 1995, ApJ, 444, 770

Nordh, L., Olofsson, G., Abergel, A., et al. 1996, A\&A, 315, L185

Paatero, P. 1999, J. Comp. Graph. Stat., 8(4), 854

Padoan, P., Juvela, M., Bally, J., et al. 1998, ApJ, 504, 300

Pfau, W., Hoff, W., \& Relke, H. 1996, MPE Report, 263, 59

Pfau, W. 2000, Priv. Comm., 482, 334

Schwartz, R. D. 1991, in Low mass star formation in southern molecular clouds, ed. B. Reipurth, ESO Sc. Report, 11, 93

Shibata, K., \& Matsumoto, R. 1991, Nature, 353, 633

Talbi, D., \& Herbst, E. 1998, A\&A, 333, 1007

Ushida, Y., Fukui, Y., Minoshima, Y., et al. 1991, Nature, 349 140

Vallée, J. P. 2000, ApJ, 538, 226

Verschuur, G. L. 1991, Ap\&SS, 185, 137

Verschuur, G. L. 1995, Ap\&SS, 227, 187

Vilas-Boas, J. W. S., Myers, P. C., \& Fuller, G. A. 1994, ApJ, 433, 96

Whittet , D. C. B., Prusti, T., Franco, G. A. P., et al. 1997, A\&A, 327, 1194

Williams, J. P., De Geus, E. J., \& Blitz, L. 1994, ApJ, 428, 693 